\title{
Ocular Swelling after Forehead Fat Graft
}

\section{Jae Woo Park}

Park Jae Woo Plastic Surgery Clinic, Seoul, Korea
No potential conflict of interest relevant to this article was reported.
Background Facial fat grafting is a common cosmetic surgery for facial contouring. Although it is considered a very safe procedure, there are several reports about complications after fat grafting. Eye complications are very rare, but do exist. The author has treated cases of eyelid swelling after forehead fat grafting with conservative or surgical treatment.

Methods From February 2010 to April 2014, the author treated five women (mean age, 35 years; range 28-52), who developed eyelid swelling (both, $n=3$; unilateral, $\mathrm{n}=2$ ) approximately 2 weeks after forehead fat grafting. Symptoms included pain, foreign body sensation, recurrent swelling and redness, unilateral or bilateral ptosis, and palpable lumps. Three were acute cases treated within 1 month, and the other two were chronic cases, which occurred 4 and 28 months after the fat graft.

Results Conservative treatment (Lasix, antibiotics, steroids) was used for two patients. The other three patients were treated with direct fat extraction or lump excision through the double eyelid crease line. Histological examination identified the excised specimens as lipocysts or liopogranulomas. All patients fully recovered, despite transient ptosis for several days immediately after the operation.

Conclusions Eyelid swelling after forehead fat graft can be successfully treated with either conservative or surgical treatment. Although few studies have reported eyelid swelling after fat grafting, this complication may become more common, in the future, as facial fat grafting becomes more popular. This treatment modality should be helpful to the plastic surgeons faced with these unpleasant complications.

Keywords Fat necrosis, Fat tissue, Forehead, Foreign body granuloma, Postoperative complications, Transplantation

\section{INTRODUCTION}

In 1912, the first known fat grafts were performed in two facial lipoatrophy patients by Eugene Hollander [1]. Liposuction had become quite popular after the introduction of the Illouz method, and fat grafting developed along with it $[2,3]$. However, fat grafting showed unpredictable results before Coleman developed the structural fat graft in $2001[4,5]$, which strongly influenced the de-

Received: May 23, 2014 Revised: Jun 18, 2014 Accepted: Jun 19, 2014 Correspondence: Jae Woo Park Park Jae Woo Plastic Surgery Clinic, 605 Gangnamdae-ro, Soecho-gu, Seoul 137-903, Korea.

E-mail: psparkjw@naver.com

Copyright @ 2014 The Korean Society for Aesthetic Plastic Surgery.

This is an Open Access article distributed under the terms of the Creative Commons Attribution Non-Commercial License (http://creativecommons.org/licenses/by-nc/3.0/) which permits unrestricted non-commercial use, distribution, and reproduction in any medium, provided the original work is properly cited. www.e-aaps.org velopment of modern fat graft surgery by improving fat harvesting methods, fat refinement, and fat placement for better long-term survival and volume replacement.

Fat may be the ideal filler material in reconstructive or cosmetic surgery. Harvesting the patient's own fat can provide sufficient quantities, and the fat is biocompatible, with no immune reaction after transplantation. For that reason, fat grafting, as a primary or adjuvant procedure, has become one of most common plastic surgeries. The best results are obtained by fat harvesting using small cannulas, by preinjection-fat preparation by centrifugation, and by the latest developments for fat placement into the tissue and postoperative care [6-8].

As fat grafting has become more commonplace, so have complications such as infections, surface irregularities, palpable masses, and unfavorable results due to poor planning. However, eyerelated complications are not common. Blindness due to ophthalmic artery occlusion after fat grafting is usually reported, because 
it is a serious condition; however, few reports regarding other eyerelated complications have been published.

The author has successfully treated several patients with acute or chronic ocular swelling after forehead fat grafting and suggests treatment modalities for similar cases.

\section{METHODS}

\section{Patients}

From February 2010 to April 2014, five women (mean age, 35 years; range, 28-52) who experienced eyelid swelling after forehead fat grafting received conservative or surgical treatment. Three cases involved both eyelids, and two cases involved only one eyelid. All patients had undergone forehead fat grafting with cryopreserved fat grafts in two cases that required surgical treatment. The eyelid swelling began approximately 5 to 7 days after the operation. The patients described intermittent eyelid swelling with heat and dull pain, which became more severe and persistent with symptoms such as foreign body sensation, redness, unilateral or bilateral ptosis, palpable lumps, and difficulty in opening the eyes, which occurred both day and night. Three cases were acute, occurring within 1 month after the fat grafting procedure, whereas two cases were chronic, occurring 4 months and 28 months after fat grafting.

\section{Conservative treatment}

All three patients with acute swelling received conservative treatment initiated the day symptoms developed, but one patient later required surgical intervention. If marked swelling of the eyes occurs after forehead fat grafting, positional drainage with bed rest may be recommended. Swelling may be worsened by gravity when sitting in an upright position or standing. In addition, ice should be used to cool the area around eyes. If no improvement occurs with 1 or 2 days, a short course of a diuretic or corticosteroid (2-3 days) may reduce the swelling.

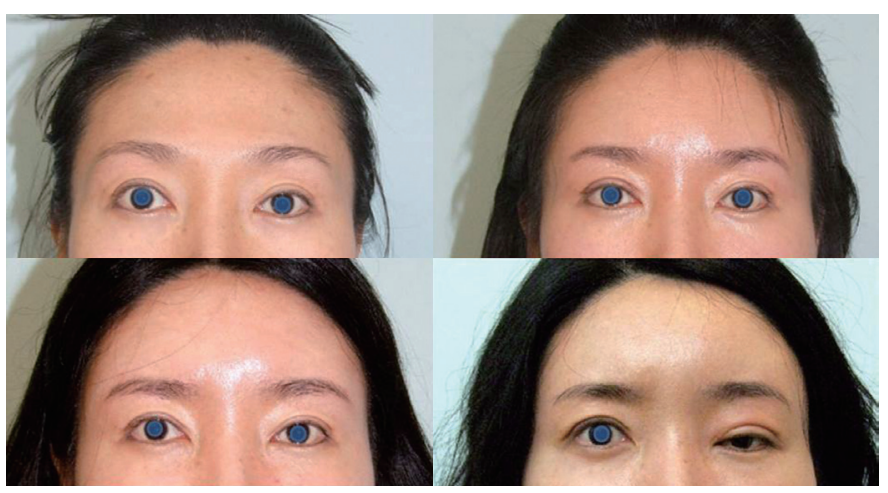

Fig. 1. She had preexisting abnormal eye swelling before fat graft. It have been more serious on next operation time at forehead fat graft combined with mass effect or ptosis (Courtesy of SM Kim).

\section{Surgical treatments}

If no improvement is observed after a week of conservative treatment, surgical exploration should be considered and performed within 30 days after the operation. Simple swelling of the eyelid usually subsides within 2 weeks. However, eyelid swelling is abnormal if it lasts more than 3 weeks, and after 4 weeks, inflammatory fibrosis results in scars. This abnormal swelling may never subside spontaneously but will likely develop into intermittent swelling or mass formation. In addition, preexisting swelling can result in more severe swelling after forehead fat grafting, leading to masses or ptosis (Fig. 1).

Before surgery, a photograph should be taken in a standard position to determine the optimal incision site. The incision line (approximately $1 \mathrm{~cm}$ long) is usually placed at the lateral one-third of the double eyelid crease (Fig. 2). The orbital septum opens easily at this location, because the abnormal swelling or mass is usually located near the intra-orbital fat in the septum. Immediately after the grafting procedure, the vacuoles will contain yellowish free oil, which becomes whitish emulsified fat over time. If more than a year has passed, a lump or mass will form in the intra-orbital fat, which should be excised. The lesions are usually located in the
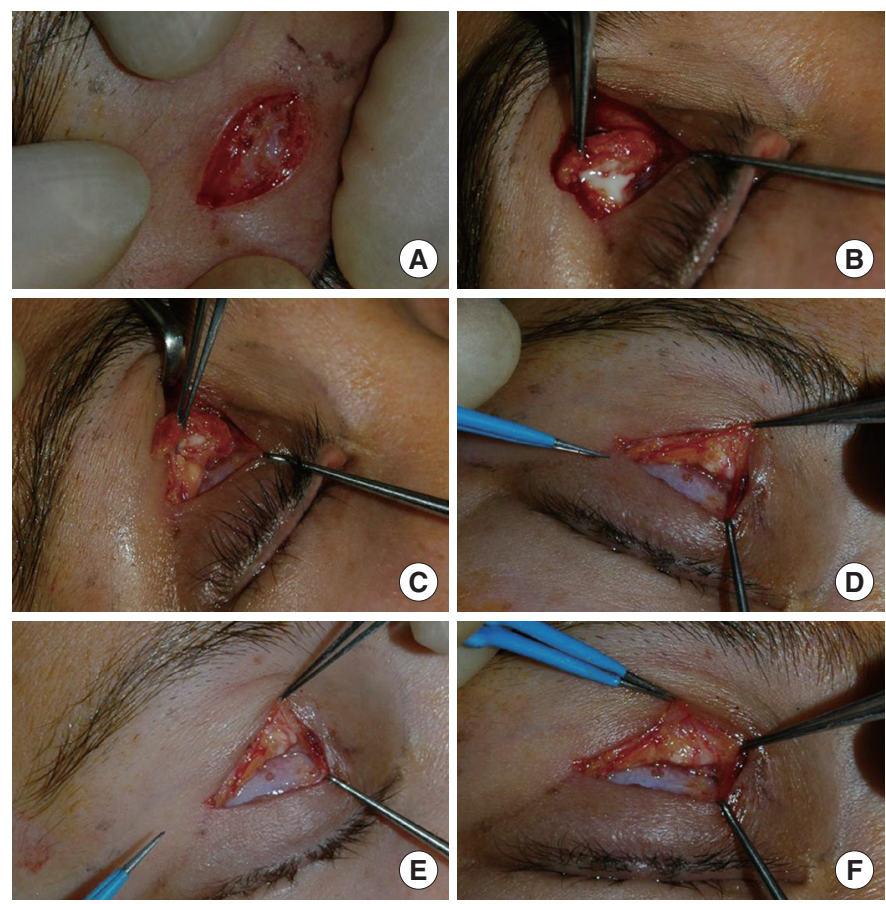

Fig. 2. Surgical treatment for the eye swelling. Each lesion is separated individually as a small sac, cause of that, you have to make it rupture to extract the free oil with the electrocautery. (A) The incision line is usually placed at the lateral one third on the double eyelid crease about $1 \mathrm{~cm}$ in length. (B) Vacuole or sacs contained with yellowish free oil or whitish emulsified fat in it. (C) The lesions are usually located lower portion of intra-orbital fat near the levator sheath. (D) It should be carefully done with gentle traction of orbital fat from anterior to posterial. (E, F) Medial intra-orbital fat lobules. 
lower portion of the intra-orbital fat, near the levator sheath. Each lesion is a small individual sac, which must be ruptured to extract the oil by electrocautery. This procedure should be performed carefully, with gentle traction of the orbital fat from lateral to middle and medial intra-orbital fat lobules (Fig. 2). If the incision is made too lateral to approach the medial fat lobule, it may be elongated for a more medial position. If the swelling is located medially, it is better to make another medial incision (approximately 1 $\mathrm{cm}$ ), with the intervening skin left intact to preserve the original eyelid crease, rather than a single long incision from lateral to medial. After removing all sacs containing free oil, the surgical field must be washed with sterile saline (with or without antibiotics), and the incision closed with 7-0 black nylon sutures.

If the lesion is left too long after the fat grafting procedure, it may become a mass that requires direct excision (Fig. 3). This mass lesion will also be located in the lower portion of the intraorbital fat, extending into the intra-orbital portion just above levator sheath. This location may explain the ptosis that appears with eyelid swelling. The mass may be attached to the levator, moving
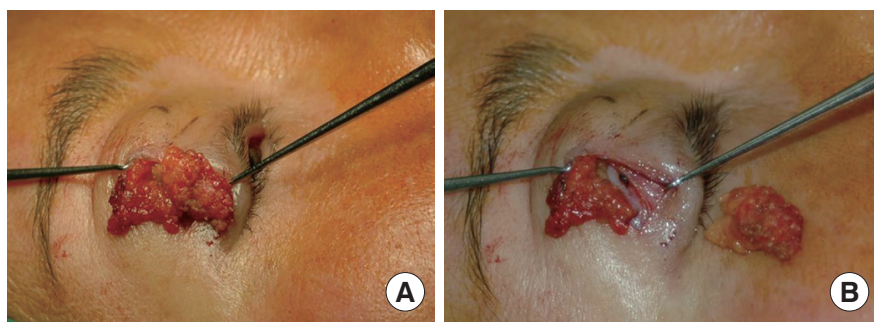

Fig. 3. The mass lesion in chronic case that should be needed a direct excision. (A) This mass lesion is also located at lower portion of the intra-orbital fat and could be separated with upper intact fat tissue. (B) The mass is extended into the intra-orbital portion just over levator sheath.
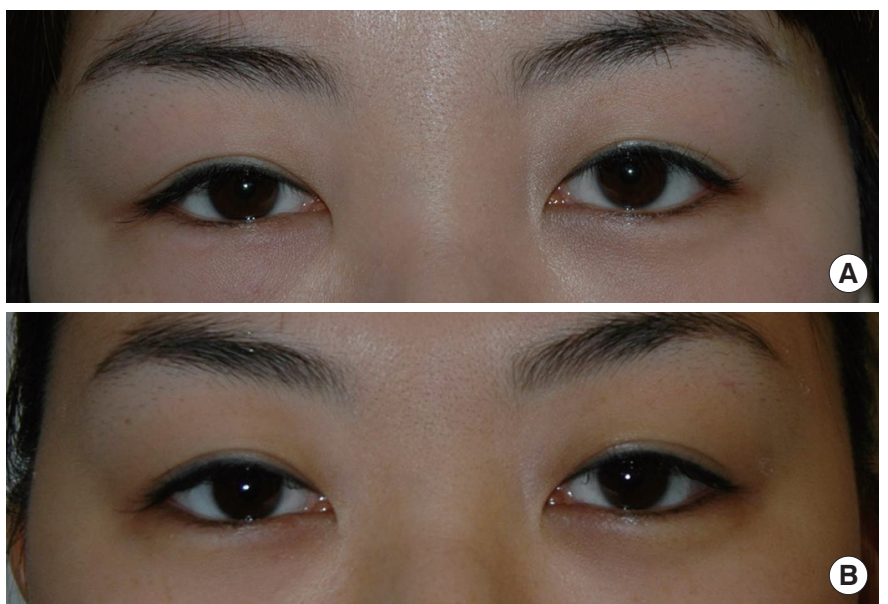

Fig. 4. Case 1. (A) A 29 years old woman complained with mild persistent eye swelling and mild ptosis on right side eye after forehead fat graft. (B) The symptom was subsided without any residual complication with conservative treatment. along with the levator. A large mass may make it difficult to open the eyes because of the mass effect.

\section{RESULTS}

Symptoms experienced by these five patients included marked eyelid swelling, dull pain without improvement after changing positions, and ptosis. Although the main lesion appeared to be subcutaneous or intramuscular, it was located in the intra-ocular fat, especially the lower portion parallel to the levator. In acute cases, this region was edematous and enlarged, with abnormal vacuoles containing free oil. The vacuole contents were oily, yellowish or milky, and without odor. The consistency was soft to hard, depending on the duration of the symptoms.

\section{Case 1}

A 29-year-old woman complained of mild but persistent eye swelling and ptosis of the right eye after forehead fat grafting (Fig. 4). After 3 weeks, the symptoms were treated with a diuretic (furosemide, $40 \mathrm{mg}$ for 2 days), a corticosteroid (prednisolone, 20 $\mathrm{mg}$ for 5 days), and an antibiotic (cefmetazole, $500 \mathrm{mg}$ twice per
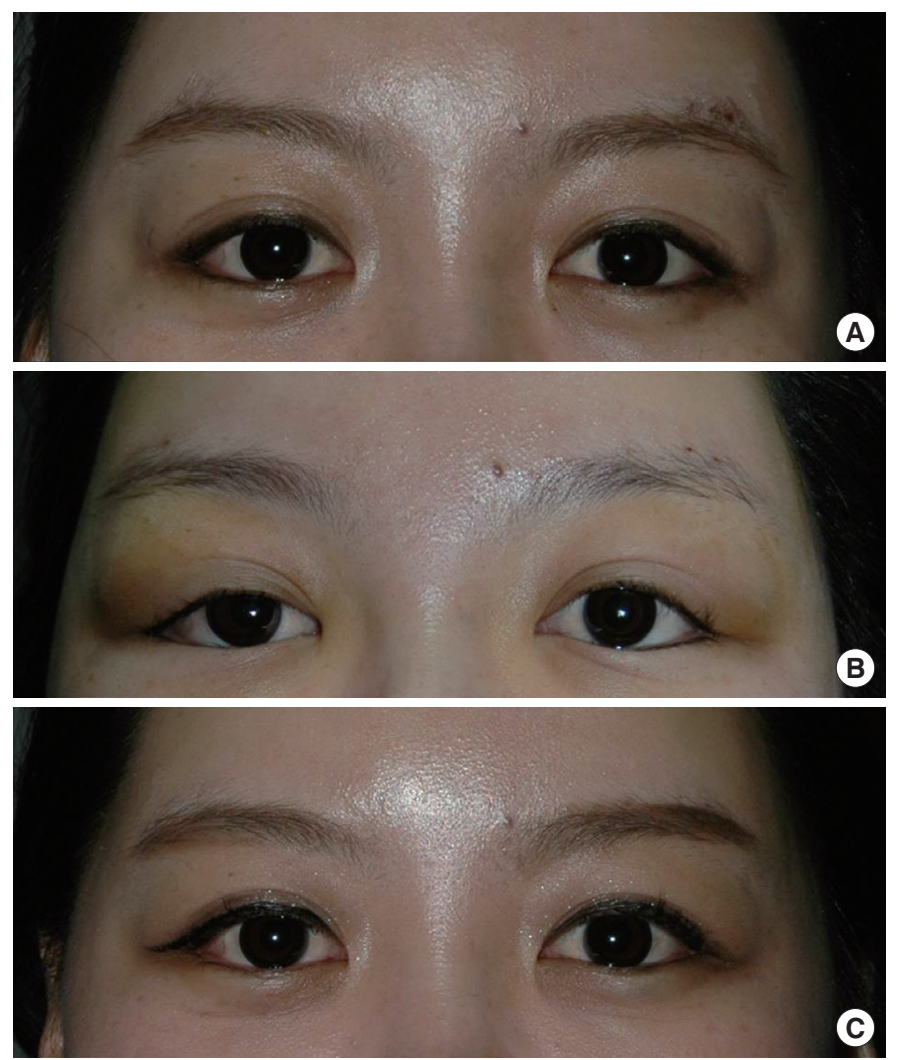

Fig. 5. Case 2. (A) A 32 years old woman had normal eyes on both side 3 days after forehead fat graft. (B) The remark periorbital swelling developed about 5 days after operation and is more aggravated in following few days on both sides. (C) It had been treated with the conservative treatment without any sequela. 
day for 3 days). The symptoms subsided without any residual complications.

\section{Case 2}

A 32-year-old woman experienced marked periorbital swelling on both sides after forehead fat grafting (Fig. 5). The swelling devel-
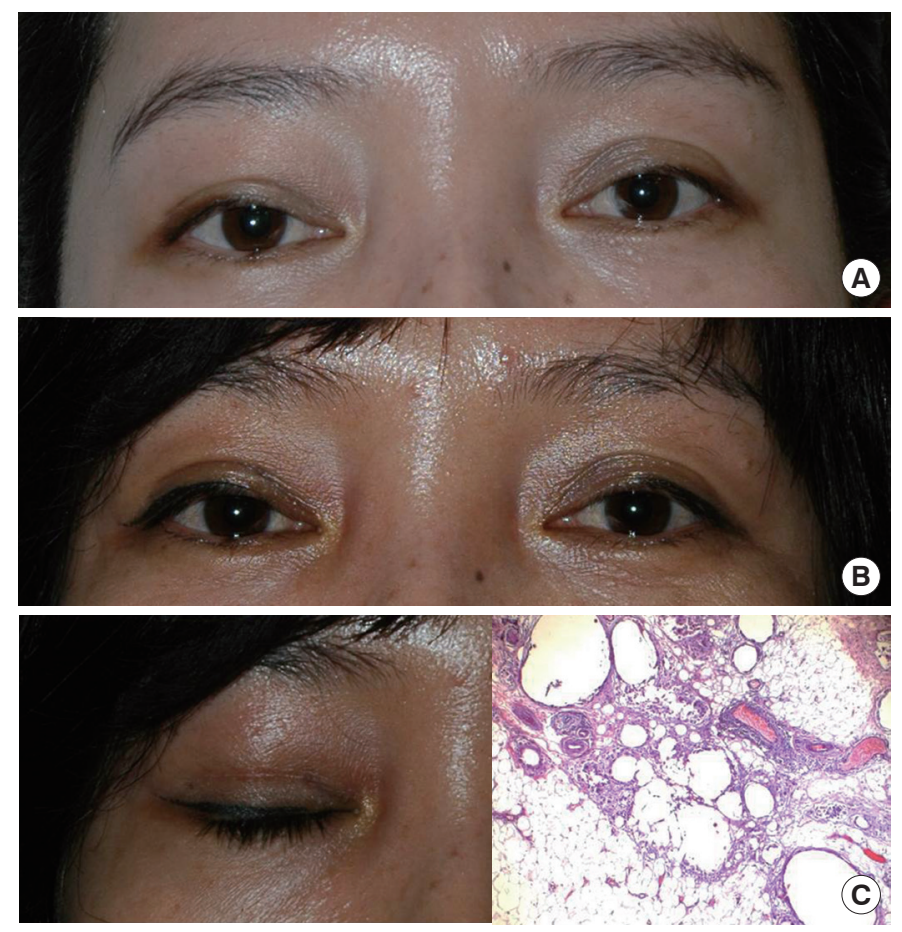

Fig. 6. Case 3. (A) A 52 years old woman complained Rt. Side eye swelling with some discomfort on eye opening. It developed about 3 weeks after operation without response or improvement with conservative treatment. (B) It was treated with surgical extraction. (C) Histologic findings showed inflammatory findings with free oil vacuoles.
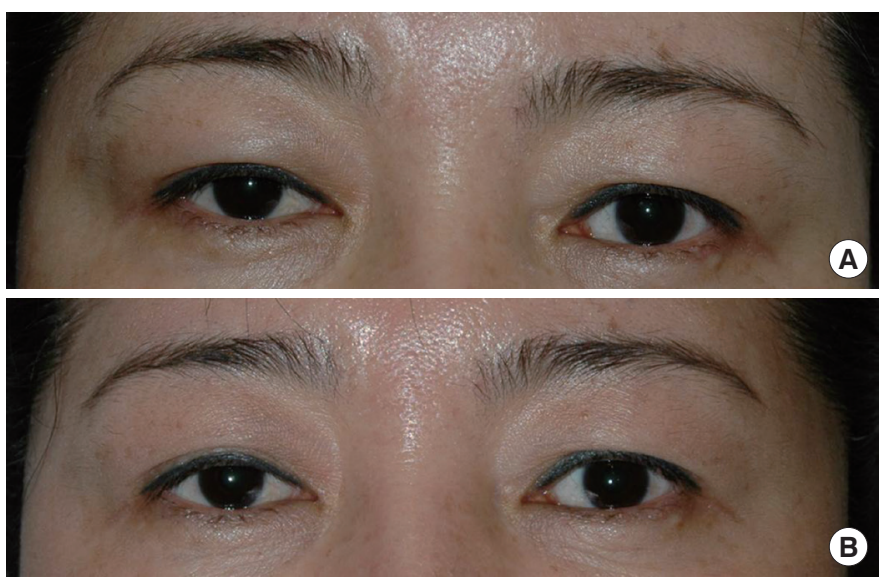

Fig. 7. Case 4. (A) A 47 years old woman complained persistent eye swelling with mild ptosis during 4 months after operation. (B) It was treated with surgical extraction of oil cyst without recurrence. The ptotic symptom was improved after surgery. oped approximately 5 days after the operation and became more severe in the following few days. Treatment was the same as that used for case 1, and the swelling resolved without any sequelae.

\section{Case 3}

A 52-year-old woman reported swelling of the right eye, with discomfort upon opening (Fig. 6). These symptoms developed approximately 3 weeks after the operation and did not improve with conservative treatment. The swelling was treated by surgical extraction of the vacuoles containing free oil. Symptoms did not recur after the surgical intervention.

\section{Case 4}

A 47-year-old woman complained of persistent eye swelling during the 4 months after forehead fat grafting, which showed only minimal response to conservative treatment (Fig. 7). The foreign body sensation worsened during follow-up. The patient was treated by surgical extraction of the oil cyst, and the swelling did not reoccur.

\section{Case 5}

A 50-year-old woman complained of recurrent eyelid swelling with dull pain that developed after forehead fat grafting, which
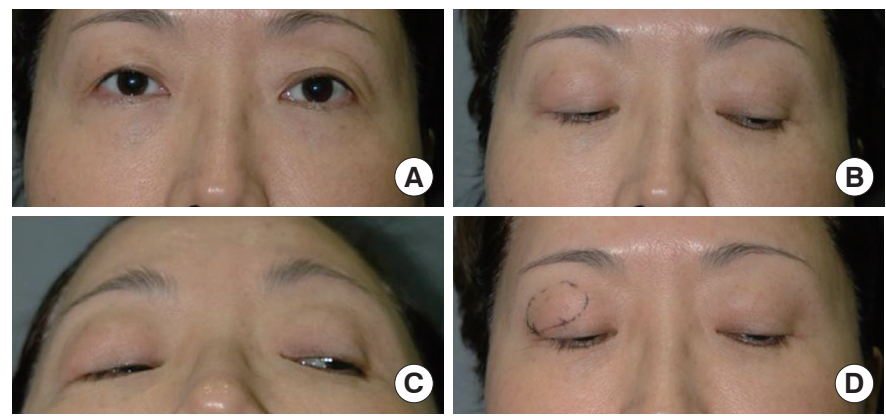

Fig. 8. Case 5. (A) A 50 years old woman complained recurrent eyelid swelling with dull pain that developed after forehead fat graft about one and half years ago. (B, C) It was accentuated on downward viewing. (D) Preoperative marking.
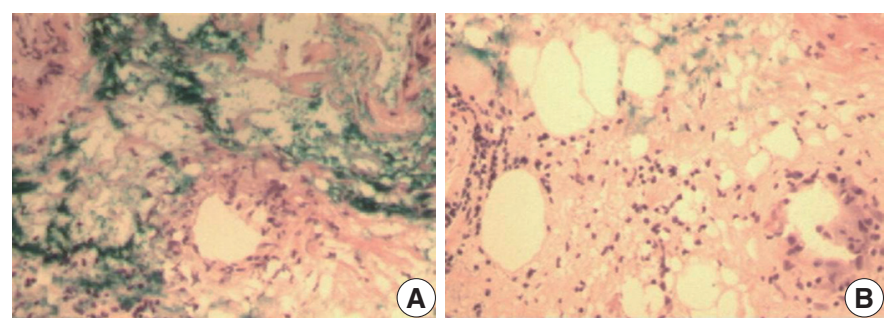

Fig. 9. (A) The histologic findings of Case 5 showed lipogranuloma. It have many inflammatory cells accumulation around many lipid filled vacuoles or sacs in variable sizesurrounded with irregular fibrosis. (B) The histologic findings of foreign body reaction to the free oilvacuoles surrounded with chronic inflammatory cells. 
was performed approximately one and a half years previously (Fig. 8, 9). A soft marked swelling developed on both eyelids approximately 1 month after the operation. The swelling was minimally improved by anti-inflammatory agents and antibiotics, but did not completely resolve, recurring at irregular intervals depending on her overall condition. For example, if the patient was tired, the symptoms were more severe. The soft swelling developed into a harder mass with redness and dull pain, and the initially transient symptoms became more persistent and severe. The patient reported that she could not open her eyes when the symptoms were severe.

\section{DISCUSSION}

These days, most doctors can achieve optimal results with fat graft surgery, which has over $90 \%$ survival rate $[9,10]$.

As fat grafting has become more popular, complications have also become more common. In 2009, a task force formed by the American Society of Plastic Surgeons assessed autologous fat grafting techniques to determine their safety and efficacy [11]. The task force made specific recommendations regarding harvest techniques, graft preparation, injection techniques, injection sites, graft storage, and use of epinephrine and lidocaine at the donor site to optimize fat graft viability. They reported that fat grafting is safe for a variety of medical conditions, with a lower risk of complications than other types of surgery. Potential risks associated with fat grafting are anesthesia-related complications, infection, bleeding, poor outcome, interference with breast cancer detection, and unexpected, life-threatening complications such as fat embolism, stroke, lipoid meningitis, and septic shock. However, eye-related complications were not mentioned. Complications involving the eye are not common after fat grafting. Although blindness caused by ophthalmic artery occlusion after cosmetic filler injection or other eye surgery has been reported [12-15], few reports have described eyelid-associated complications [16].

Despite the development of blunt-tipped cannulae some doctors still use sharp cannulae, which are more likely to pierce vessels, resulting in a fat embolism. Fat grafts accidentally injected into the facial artery or its branches, supraorbital artery, or supratrochlear artery may cause serious problems such as partial or total blindness, or tissue necrosis around the eyes or nose $[13,14]$ Even with a blunt-tipped cannula, these complications could occur causing vascular damage during pre-tunneling procedures or high-volume or high-pressure injections into a small area. The cannula should be gently inserted, and the fat slowly injected while withdrawing the needle to prevent blindness after facial fat grafting.

Periorbital swelling after facial fat grafting is underestimated because of failure to adequately examine patients. Although physiological swelling is a typical finding after fat grafting and gradually subsides, pathological periorbital swelling occurred in the five cases presented here. The patients experienced marked orbital swelling, beginning 5 to 7 days after the operation. The swelling was intermittent, with heat and dull pain that did not decrease with positional changes or time. The symptoms worsened at times, making it difficult to open the eyes. Ptosis often occurred on one or both sides.

Mechanisms underlying ocular swelling after forehead fat grafting include oil leaking from the forehead into the periocular area through the supraorbital or supratrochlear foramen. The method of fat placement is an important consideration. For successful fat grafting, Coleman suggested diffuse infiltration with multiple passes, placing extremely small amounts of fat with each pass, and separating the newly grafted fat parcels from each other. This technique ensures a large area of contact between capillaries and the grafted fat, promoting nutrition and respiration $[4,5]$ However, many rapid strokes of the cannula can produce bleeding and edema, with increased pressure in the grafted area and decreased circulation. As a result, more fat cells rupture, and the free oil that leaks into the forehead can move to the ocular area through the supraorbital or supratrochlear foramen, especially if the foramen is wide or not closed (i.e., notch), free oil is more likely to leak into the periocular area.

Another possible explanation for orbital swelling after forehead fat grafting is weakening of the orbicularis retaining ligament, which is attached to the periosteum approximately $2-3 \mathrm{~mm}$ above the superior orbital rim. Normally, this acts as a barrier between the forehead and orbital area to prevent the spread of infection to the orbital area. Injecting material below or crossing this ligament can result in intraocular placement [13]. Infiltration during surgery or swelling afterward can weaken this structure, allowing free oil to leak into the orbit.

Finally, orbital swelling can occur through excessive retro-orbicularis oculi fat pads, which may extend over the orbital septum, allowing free oil to leak through the spaces between them and spread into the orbit.

Free oil can spread to the lower half of the orbital fat over the levator sheath due to gravity. Surgical findings of cases 3, 4, and 5 showed that the lesions were vacuoles containing free oil or hard masses in the orbital fat. Histologic findings for all cases revealed the accumulation of inflammatory cells around numerous lipidfilled vacuoles of various sizes, with some fibrosis (Fig. 9). In a case of chronic swelling, the histology results revealed a foreign body reaction to the free oil. Cryopreserved fat was used in cases 3 and 4 , which may have been the cause of ocular swelling. Cryopreserved fat is fragile, with a higher percentage of free oil compared with freshly harvested fat; therefore, the fat cells are more likely to rupture and leak free oil. For that reason, cryopreserved fat must be centrifuged again ( $>3,000 \mathrm{rpm}$ for $5 \mathrm{~min}$ ). In addition, cryopreserved fat are more likely to become contaminated with bacteria or fungi. Contamination can occur during process- 
ing (e.g., harvesting, centrifugation) and microorganisms can grow at low storage temperatures and anaerobic conditions. Even lowvirulence microorganisms can cause chronic infections after fat grafting in this ideal growth environment; therefore, persistent ocular swelling can indicate a weak immune system. There was some response to antibiotic and steroid therapy, indicating the possibility of infection with low-virulence microorganisms.

All patients reported ptosis on one or both sides. Ptosis after fat grafting is related to free oil-containing vacuoles located just above the levator sheath. In case 5 , a hard lump $(1 \times 1 \times 0.5 \mathrm{~cm})$ was located in the lower half of the intra-orbital fat lobules on the lateral side, just above the levator. It was separated from the levator tendon by the levator sheath and fat lobule sheath, and attached to these sheaths by an inflammatory adhesion, preventing the eye from opening completely and causing ptosis. A mass adhering to the levator tendon moves along with the levator oculi but is checked by the transverse ligament or other intraocular structures. If ptosis was present before surgery, it may worsen with ocular swelling after fat grafting. This type of ptosis may become more severe with successive forehead fat grafting.

Conservative treatment may be helpful for acute ocular swelling after forehead fat grafting. This includes watchful waiting, cooling the eyes with ice, anti-inflammatory drugs, steroids, diuretics, and sometimes antibiotics. Minimal swelling typically subsides with ice and positional treatment; lying in a supine position allows the free oil to drain into the scalp area or other facial areas. Weakened orbicularis oculi ligaments may resume the normal barrier function with anti-inflammatory drugs, steroids, and diuretics, which reduce the inflammatory swelling around this ligament. Antibiotics are helpful if a subclinical infection contributes to the swelling. If symptoms do not respond or worsen with conservative treatment, surgical treatment may be considered. Lesions were treated by direct removal of the encapsulated emulsified or free oil in cases 3 and 4 , and by direct excision of the mass adherent to the surrounding tissues without encapsulation.

Paik et al. [16] reported two cases of unusual unilateral eyelid swelling with multiple small lumps. The patients had undergone autogenous fat injection for cosmetic forehead augmentation approximately 6 months and 9 months previously. The small (diameter, 5-10 $\mathrm{mm}$ ), palpable, hard, nonmobile lumps were evaluated by magnetic resonance imaging (MRI). All masses were situated deep in the tissue, near the superior or lateral orbital rim, which were deeper than the cases presented in the present study. Histological findings revealed foreign body lipogranuloma, chronic inflammation, and fibrosis, similar to the cases in the present study. Treatment consisted of excision of all masses to relieve symptoms (e.g., swelling, moderate ptosis, lagophthalmos) [16]. The authors did not use conservative treatment in these chronic cases but suggested that conservative treatment may be effective in the acute stage ( $<1$ month). Although, Paik et al. described lesions near the superior or lateral orbital rim, they would appear the same on MRI as the lesions described in the present case series. Making the incision on the lateral one-third of the natural double eyelid crease allows easy access to the lateral portion of the orbital fat. In the present case series, the lesions appeared to involve the skin or muscle over the orbital septum; however, surgery revealed that the main lesion involved intraocular fat, primarily in the lower portion parallel to the levator.

In summary, the author describes the successful treatment of five patients who experienced ocular swelling of varying duration after forehead fat grafting. Cases involving previous ptosis require ruling out diseases or drugs as the cause of ptosis before surgery. Some cases of ocular swelling may be related to the use of cryopreserved fat; therefore, fresh fat should be used if possible. Injecting excessive amounts of fat into the forehead at one time is not recommended. In acute cases of ocular swelling after fat grafting, conservative treatment is recommended, followed by surgical treatment, if necessary.

\section{REFERENCES}

1. Joseph M. Handbuch der kosmetik. Leipzig: Veit \& Co.; 1912. p. 690-1.

2. Illouz YG. The fat cell "graft": a new technique to fill depressions. Plast Reconstr Surg 1986;78:122-3.

3. Illouz YG. Present results of fat injection. Aesthetic Plast Surg 1988;12: 175-81.

4. Coleman SR. Structural fat grafts: the ideal filler? Clin Plast Surg 2001; 28:111-9.

5. Coleman SR. Structural fat grafting: more than a permanent filler. Plast Reconstr Surg 2006;118:108-20.

6. Guyuron B, Majzoub, RK. Facial augmentation with core fat graft: a preliminary report. Plast Reconstr Surg 2007;120:295-302.

7. Sommer G, Sattler, G. Current concepts of fat graft survival: histology of aspirated adipose tissue and review of the literatures. Dermatol Surg 2000;26:1159-66.

8. Butterwick KJ. Lipoaugmentation for aging hands: a comparison of the longevity and aesthetic results of centrifuged versus noncentrifuged fat. Dermatol Surg 2002;28:987-91.

9. von Heimburg D, Hemmrich K, Haydarlioglu S, et al. Comparison of viable cell yield from excised versus aspirated adipose tissue. Cells Tissues Organs 2004;178:87-92.

10. Coleman, SR. Hand rejuvenation with structural fat grafting. Plast Reconstr Surg 2002;110:1731-44.

11. Gutowski KA. Current applications and safety of autologous fat grafts: a report of the ASPS Fat Graft Task Force. Plast Reconstr Surg 2009; 124:272-80.

12. Lazzeri D, Agostini T, Figus M, et al. Blindness following cosmetic injections of the face. Plast Reconstr Surg 2012;129:995-1012.

13. Park SW, Woo SJ, Park KH, et al. Iatrogenic retinal artery occlusion caused by cosmetic facial filler injections. Am J Ophthamol 2012;154: 


\section{3-62.}

14. Kim KE, Ahn SJ, Woo SJ, et al. Central retinal artery occlusion caused by fat embolism following endoscopic sinus surgery. J Neuroophthalmol 2013;33:149-50.

15. Alishiri A, Naderi M, Jadidi K, et al. Sudden unilateral visual loss and ptosis after autologous fat injection and simultaneous Bont/A treatment. J Clin Case Rep 2014;4:3.

16. Paik JS, Cho WK, Park GS, et al. Eyelid-associated complications after autogenous fat injection for cosmetic forehead augmentation. BMC Ophthalmol 2013;13:32. 